\title{
Aculturación, involucramiento parental y autoeficacia materna: Un es- tudio cualitativo con mujeres latinoamericanas en los Estados Unidos
}

\section{Acculturation, parental involvement, and maternal self-efficacy: A qua- litative study among Latin American women in the United States}

\author{
Arturo Enrique Orozco Vargas*
}

\begin{abstract}
${ }^{*} \mathrm{PhD}$ en Investigación Educativa con una concentración en Desarrollo Humano y Estudios de la Familia. Investigador y Profesor de tiempo completo en la Licenciatura en Psicología en el Centro Universitario UAEM Atlacomulco. E-Mail: dr.enrique.orozco@hotmail.com
\end{abstract}

Centro Universitario UAEM. Atlacomulco, México.

\section{Resumen}

La inmigración a los Estados Unidos conlleva para las familias latinoamericanas una serie de desafíos y adversidades así como múltiples oportunidades y beneficios. Desde esta perspectiva, el objetivo de la investigación que se informa fue analizar el impacto que tiene este proceso de adaptación en el involucramiento parental y la auto-eficacia materna de las mujeres inmigrantes latinoamericanas que residen en los Estados Unidos. En este estudio participaron 14 mujeres provenientes de cinco países latinoamericanos. Los principales hallazgos muestran que las participantes tuvieron que adaptarse a las tradiciones, leyes y normas de convivencia imperantes en los Estados Unidos. Con respecto a sus expectativas, las participantes describieron el deseo de obtener un trabajo bien remunerado para poder satisfacer las necesidades básicas de su familia así como proveer a sus hijos de una buena educación. Entre las principales adversidades se encuentran el idioma, el estatus migratorio y la separación de sus familiares. En contraste, diversos beneficios tales como la educación de los hijos y la información profesional que recibieron han sido determinantes en su involucramiento parental. De la misma manera, las participantes enfatizaron la importancia de diversos recursos que han encontrado en el país de residencia entre los que se encuentran el apoyo recibido del gobierno norteamericano, los cursos en los que han participado y el apoyo social brindado por otras familias latinoamericanas. Los resultados de la investigación permiten concluir que a pesar de los muchos obstáculos a los que se enfrentan, todas las participantes mencionaron contundentemente que el haber inmigrado a los Estados Unidos ha enriquecido su experiencia como madres.

Palabras clave: Aculturación; Autoeficacia materna; Involucramiento parental; Inmigrantes; Latinoamericanos; Mujeres.

\begin{abstract}
The process of immigration involves multiple challenges and barriers as well as benefits and opportunities. Each year, innumerable Latin American families immigrate to the United States of America seeking employment, medical care, or education for their children. The purpose of this qualitative research was to explore the effects of the process of acculturation in the parental involvement and maternal self-efficacy among Latin American immigrant mothers living in the United States. In order to achieve this objective, four research questions were formulated: (1) What is the description that Latin American immigrant women express about their own process of
\end{abstract}


acculturation? (2) What are the changes these women have experienced during their acculturation process to the United States of America? (3) How Latin American immigrant mothers are involved in the academic, emotional, social, and behavioral development of their children (4) What are the perceptions that Latin American immigrant women have about their abilities and resources as mothers?

In the present study, 14 women from five different Latin American countries participated in qualitative interviews. Participants' narratives were analyzed using the Consensual Qualitative Research method proposed by Hill, Thompson and Williams. After having analyzed all transcripts, five main themes were identified: (1) Adaptation, (2) Expectations, (3) Challenges, (4) Benefits, and (5) Resources.

Results shows that participants had to adapt their behavior and ideas to the current American traditions, laws, and norms. Regarding participants' expectations, they talked about how one of their main expectations was to obtain an employment with which they could satisfy their basic needs and provide a good educational level to their children. Immigrants experience multiple obstacles; for instance, lack of employment and health services, legal status, changes in family functioning, separation from family, discrimination, fear of deportation, intergenerational conflicts, and language proficiency. Besides these external barriers and challenges, Latin American immigrants are affected by internal challenges with possible negative consequences, such as intimate partner violence, despondent moods, loneliness, feeling of inadequacy, gender inequality, stress, anxiety, and alcoholism. In this study, the main barriers these participants had to cope were their immigration status, separation from their families, and language proficiency. In contrast, these participants had several benefits as a result of their decision to immigrate to the United States of America such as economic resources, their children's education, and professional information they received that helped them improve their maternal skills. In consequence, they have been able to develop their maternal skills and spend quality time with their children. All participants in this research emphasized that these benefits have been determinant for their parental involvement.

The last factor related to their maternal experience was the resources they have found in the United States of American. Participants talked about the support they have received from the American government, formal and informal knowledge about how to become a better mother, and the social support provided by other Latin American families. In general terms, immigrants experience better life conditions when they have a network of relatives and friends previously established in the receiving country. Consequently, they have more opportunities for a successful relocation in the new region. The importance of social support is related to the presence of these networks which provide any kind of support when necessary. In particular, due to this support, these participants have had a better adaptation to their new life. In conclusion, this research reveals that in spite of multiple challenges Latin American immigrants have to cope, all participants concluded that after immigrating to the United States of America their competence and involvement as mothers have increased.

Key words: Acculturation; Maternal self-efficacy; Parental involvement; Immigrants; Latin Americans; Women.

\section{Introducción}

Durante las dos últimas décadas, los latinoamericanos han constituido el grupo étnico minoritario más numeroso que vive en los Estados Unidos, alcanzando los $55 \mathrm{mi}-$ llones de latinoamericanos en el 2014 (US Census Bureau, 2015). Estimaciones hechas por el Pew Research Center (2008) señalan que para el año 2050 la población latinoamericana llegará a los 127 millones. El impacto de los latinoamericanos en los Estados Unidos ha quedado plasmado a través de los siglos en su música, tradiciones, literatura, arquitectura, gastronomía, lenguaje y educación, entre otros. Por el contrario, la comunidad latinoamericana también se ve influenciada permanentemente por la interacción con los ciudadanos estadouniden- 
ses. Como resultado de este intercambio mutuo surge un fenómeno sociocultural denominado aculturación. De acuerdo con Berry (1997), este término se refiere al proceso cultural y psicológico que se lleva a cabo como resultado del contacto mutuo entre dos o más grupos culturales. Grupalmente este proceso conlleva cambios en las estructuras sociales, en las instituciones y en las prácticas culturales e individualmente, la aculturación involucra cambios en la personalidad y el comportamiento de los miembros de la sociedad.

A inicios del Siglo XX, diversos investigadores comenzaron a estudiar el fenómeno de la aculturación desde múltiples perspectivas. El constante flujo de migrantes hacia los Estados Unidos creció de tal manera que para la década del 30, Redfield, Linton y Herskovits (1936) aplicaron el término aculturación para referirse a las interacciones de primera mano que establecen grupos de personas provenientes de diferentes culturas. Estudios posteriores han analizado las causas, factores de riesgo, motivaciones, consecuencias, expectativas y beneficios que conlleva la aculturación. Para explicar el proceso de aculturación se han propuesto diversos modelos, entre los que se destacan los dimensionales que ofrecen una descripción comprensiva acerca de la adaptación sociocultural y psicológica de los inmigrantes.

El más representativo de estos modelos fue el desarrollado por Berry, quien afirmó que la adaptación a una nueva cultura es para muchos inmigrantes un gran desafío (1980). Este modelo incluye cuatro principales estrategias de aculturación (Asimilación, Separación, Marginalización e Integración) derivadas de dos orientaciones básicas. La primera orientación es una preferencia relativa que los individuos mantienen con respecto a su propia identidad y herencia cultural. La segunda es una preferencia relativa para participar y tener contacto con otros grupos etnoculturales que están viviendo en la sociedad (Berry, 2001). Aunque es considerado uno de los modelos más importante en el estudio de la aculturación, éste no se encuentra exento de críticas. Uno de los principales cuestionamientos que se le han hecho a este modelo, es la ausencia de un análisis exhaustivo que permita explicar el impacto que tiene la sociedad de acogida en el proceso de adaptación que viven los migrantes, como así también en los cambios que experimentan los migrantes una vez que llegan a un nuevo país y a lo largo de su permanencia en él. Por otra parte, el modelo de Berry no describe las enormes diferencias que se presentan en lo que respecta a los recursos que tienen los migrantes en comparación con los que poseen los residentes del país de acogida, lo cual conduce a relaciones de poder desiguales entre los diversos grupos étnicos (Gónzalez-Rábago, 2014).

Otra de las limitaciones que han sido señaladas reiteradamente es la descripción estática que hace Berry del proceso de aculturación. Por ejemplo, a diferencia del modelo propuesto por LaFramboise, Coleman y Gerton (1993), que analiza la aculturación como un proceso dinámico, el modelo de Berry no contempla este dinamismo al examinar la participación de los agentes y las relaciones que se establecen en el proceso de aculturación. Finalmente, el modelo propuesto por Berry ha sido criticado por no atribuir la suficiente importancia a los efectos que conllevan las relaciones interpersonales entre los miembros de los diversos grupos étnicos que conviven en el país receptor (Bourhis \& Monteuil, 2001).

Estudios realizados en todo el mundo han documentado los múltiples efectos que tiene el proceso de aculturación en la vida de los migrantes (e.g., Bacallao \& Smokowski, 2007; Brown, Langille, Tanner \& Asbridge, 2014; Lawton \& Gerdes, 2014; Marsiglia, Nagoshi, Parsai \& Castro, 2014; Tran, Jorm, Johnson, Bambrick \& Lujic, 2015). Una de las áreas que no se encuentra exenta de su influencia, es la familia. A pesar de la importancia que tiene la familia para los latinoamericanos, son muy pocos los estudios que han investigado el impacto de la aculturación en el entorno familiar. Uno de estos estudios analizó la relación 
que existe entre los esfuerzos que hacen los padres para compartir con sus hijos las tradiciones y costumbres de sus países de origen y la manera en que sus hijos las preservan particularmente durante la adolescencia. Los hallazgos mostraron que los adolescentes que más se apegan a los valores tradicionales de sus padres exhiben un mayor compromiso y pertenencia a su grupo étnico (Umaña-Taylor, Bhanot \& Shin, 2006). Por otra parte, Gutmann (2003) encontró en un estudio con latinoamericanos, que el proceso de aculturación modifica gradualmente la participación del padre en las actividades del hogar llegando a ser más equitativo con su pareja en lo que respecta al cuidado de los hijos. Asimismo, los padres latinoamericanos con un nivel de aculturación más alto tienden a usar más argumentos verbales cuando se trata de modificar el mal comportamiento de los hijos, evitando de esta manera el uso de castigos corporales y reprimendas (Fontes, 2002).

Además de la convivencia, la herencia cultural, la mutua colaboración y la disciplina, uno de los factores de mayor impacto en el crecimiento integral de los hijos es el involucramiento parental, particularmente el de la madre. En la cultura latinoamericana, la mujer tiene un papel determinante en la crianza de los hijos. Siendo ellas quienes pasan la mayor parte del tiempo con los hijos, su nivel de involucramiento tiene amplias repercusiones en el bienestar de los hijos. Diversos estudios han identificado una fuerte relación entre el involucramiento parental y el desarrollo físico, psicológico y social de los niños y adolescentes (e.g., Lee, Yu \& Choi, 2012; Schleider, Vélez, Krause \& Gillham, 2014). En términos generales, el involucramiento parental está relacionado con la capacidad que tienen los padres para proveer a sus hijos un hogar, alimentación, protección, salud, educación y actividades recreativas, entre otras. De acuerdo con Finley y Schwartz (2004), el involucramiento parental se define como el compromiso, interés y vínculo parental que se gesta en diversas facetas de la vida de los hijos, entre las que se encuentran el desarrollo emocio- nal y cognoscitivo, el juego y la diversión, la convivencia social, la responsabilidad y la autonomía.

Un elemento relacionado directamente con el involucramiento de los padres con sus hijos es el concepto de autoeficacia parental. Teniendo como referente teórico los principios postulados en la teoría del aprendizaje social, el comportamiento humano tiene su fundamento en el ambiente social (Bandura, 1986). Por medio de las interacciones que establece con los miembros de la sociedad, el ser humano elabora múltiples percepciones acerca de sí mismo y de todo aquello que lo rodea. Una de ellas tiene que ver con la percepción que las personas tienen de sus propias capacidades. Bandura (1977) postuló el término autoeficacia para referirse a las creencias que cada persona tiene de su propia capacidad, las mismas que son empleadas para desarrollar una serie de conductas por medio de las cuales se consigue el cumplimiento de las metas individuales. De acuerdo con Bandura (2012), la autoeficacia conlleva no solamente el desarrollo de las capacidades cognitivas, afectivas, motivacionales y volitivas, también es un elemento clave para perseverar en la confrontación de las experiencias adversas.

Debido al impacto que ha tenido el constructo de autoeficacia se ha aplicado en distintas disciplinas del conocimiento (Sociología, Psicología, Antropología, Educación, Salud, entre otras). Durante la década de 1980 se llevaron a cabo diversas investigaciones con la finalidad de explorar los efectos de la autoeficacia en el ambiente familiar y las funciones parentales. Como consecuencia de estos estudios surgió el término autoeficacia parental, el cual se define como la percepción que tienen los padres de sus propias expectativas con respecto al alcance de sus capacidades (Jones \& Prinz, 2005). Un elemento determinante en el desarrollo de la autoeficacia parental son las creencias de los padres. Ellos las utilizan en este caso para evaluar el grado de influencia que tienen en la vida de sus hijos (Ardelt \& Eccles, 2001). Aunado a ello, los recur- 
sos individuales, los estilos parentales, las experiencias previas y los rasgos de personalidad de los hijos van conformando la forma en que los padres valoran su nivel de competencia parental.

La importancia de este estudio radica en el análisis de los efectos que tiene la aculturación en la vida de las familias latinoamericanas que viven en los Estados Unidos. Ante la falta de un conocimiento profundo del impacto que tiene la adaptación social, familiar e individual en el involucramiento de los padres, la investigación realizada buscó además de llenar este vacío en la literatura científica, describir específicamente la influencia de diversos factores culturales en el desempeño de las madres latinoamericanas que han inmigrado a los Estados Unidos. De esta manera, el conocimiento de las distintas etapas por las que pasan las madres latinoamericanas en su proceso de aculturación permitirá proponer acciones y programas encaminados a mejorar su labor como madres. Asimismo, los resultados de esta investigación servirán para identificar detalladamente los recursos y habilidades que las madres latinoamericanas emplean en la crianza de sus hijos.

Teniendo en cuenta estos factores, el objetivo de esta investigación cualitativa fue analizar el impacto que tienen el proceso de aculturación en el involucramiento parental y la autoeficacia materna de las mujeres inmigrantes latinoamericanas que residen en los Estados Unidos. Con la finalidad de profundizar en los efectos de la aculturación en el funcionamiento parental, se formularon cuatro preguntas de investigación:

¿Cuál es la descripción que las mujeres inmigrantes latinoamericanas hacen de su propio proceso de aculturación?

¿Cuáles son los cambios que ha provocado su propia aculturación en los Estados Unidos?

¿Cómo se involucran las madres inmigrantes latinoamericanas en la vida académica, emocional, social y conductual de sus hijos?

¿Cuál es la percepción que las mujeres inmigrantes latinoamericanas tienen de sus capacidades y recursos como madres?

\section{Método}

\section{Participantes}

Participaron en forma anónima 14 mujeres inmigrantes que residían en Estados Unidos. Eran cinco mexicanas, cuatro salvadoreñas, una ecuatoriana, dos peruanas y dos costarricenses. Las integrantes de la muestra decidieron voluntariamente participar en el estudio respondiendo a una entrevista semiestructurada. Para ello debían haber inmigrado por los menos 5 años antes y tener al menos un hijo en edad escolar. Su promedio de edad fue igual a 26.8 años $(D E$ $=2.10$ ). La mayoría de ellas estaban casadas (9 mujeres), tres de ellas estaban separadas de su pareja y dos eran madres solteras. El número de hijos oscilaba entre 2 y 5 , con un promedio de 2.8 hijos. Con respecto a su nivel educativo, cuatro de ellas tenían estudios de primaria, siete de secundaria, dos habían estudiado la preparatoria y uno tenía estudios concluidos de licenciatura. La participante que más años tenía como inmigrante eran 20 y la que menos años tenía eran $6(M=10.8, D E=4.44)$. Todas las participantes tenían un estatus de indocumentadas.

\section{Procedimiento}

A las autoridades del consulado mexicano en la ciudad de Dallas y de tres centros comunitarios para latinoamericanos localizados en el norte de Texas se les entregó el protocolo que había sido diseñado para este estudio y los requisitos necesarios para que las entrevistas fueran confidenciales. Después de haber conseguido las autorizaciones correspondientes, se contactó a las personas que trabajaban directamente con los inmigrantes, y ellas nos pusieron en contacto con una mujer inmigrante que reunía las características de inclusión.

Por medio del procedimiento de bola de nieve se obtuvo el total de la muestra. Una vez que fueron invitadas a participar, se explicaron a cada una los objetivos de la investigación y al mismo tiempo se les en- 
tregó un consentimiento informado. Quienes estuvieron de acuerdo en participar, lo firmaron.

Las entrevistas se realizaron en el consulado y en los centros comunitarios en un lugar, donde las participantes se sintieron cómodas y seguras de la confidencialidad de sus testimonios y tuvieron una duración aproximada de una hora y media.

\section{Protocolo de la entrevista}

Con la finalidad de recoger las narrativas de las participantes, se diseñó una entrevista semiestructurada en español, la cual consistió en ocho preguntas. Estas preguntas se redactaron en base a artículos teóricos y estudios empíricos que han investigado el fenómeno de la aculturación en inmigrantes latinoamericanos así como el funcionamiento familiar que tienen los latinoamericanos residentes en Estados Unidos. Algunas de las preguntas que se incluyeron en el protocolo de entrevista son las siguientes: ¿Cómo te describirías a ti misma como madre?, ¿De qué manera te involucras en las actividades escolares de tus hijos?, ¿A qué juegas con tus hijos?, ¿Cuáles son los principales desafíos que encontraste al principio de tu estadía en los Estados Unidos?, Después de haber inmigrado a los Estados Unidos, ¿cómo has satisfecho tus expectativas familiares?

\section{Análisis de los datos}

Las narrativas de las participantes fueron analizadas usando el método de investigación cualitativo consensual (Consensual Qualitative Reseach - CQR) propuesto por Hill, Thompson y Williams (1997). Esta metodología tiene como objetivo estudiar pocos casos en profundidad usando palabras clave que describan el fenómeno a investigar, verificando los resultados al revisar sistemáticamente los datos brutos y tomando decisiones por medio de consensos.

Después de haber transcripto las entrevistas, un grupo de cuatro investigadores co- menzaron a codificar las narrativas. El primer paso consistió en identificar los dominios, los cuales sirvieron como temas generales. En el siguiente paso se clasificaron todas las narrativas en su correspondiente dominio. El objetivo del tercer paso fue identificar las ideas clave y crear con ellas las categorías que se incluirían en cada dominio. Finalmente, se reunieron los cuatro investigadores hispanohablantes que conformaban el equipo de investigación de Estudios de la Familia de una universidad norteamericana, para verificar la saturación de la información y replantear los dominios y categorías hasta obtener un acuerdo consensado. De acuerdo con la metodología $\mathrm{CQR}$, las categorías que se aplican a todos los participantes reciben el nombre de categorías generales, aquellas que se presentan en la mitad o más de las narrativas se llamarán categorías típicas y las que se presentan en menos de la mitad de los casos se clasificarán como categorías divergentes.

\section{Resultados}

Las entrevistas en profundidad permitieron conocer en detalle el proceso de aculturación que cada participante ha experimentado así como el impacto que tuvo dicho proceso en su labor como madres y en la relación con sus hijos. En el análisis de las narrativas de las 14 mujeres inmigrantes que participaron en esta investigación, se identificaron cinco temas principales. Dada la metodología empleada, estos temas generales reciben el nombre de dominios, los cuales incluyeron: (1) Adaptación, (2) Expectativas, (3) Adversidades, (4) Beneficios y (5) Recursos. En el Cuadro 1 se presentan estos dominios con sus respectivas categorías.

\section{Adaptación}

Las participantes tuvieron que realizar múltiples cambios para adaptarse de la mejor manera a su nueva vida en los Estados Unidos. La mayoría de ellas relató los problemas que tuvieron para familiarizarse con las leyes. Durante los primeros meses, aquellas que tu- 
vieron el apoyo de sus familiares o amigos comenzaron a conocer de manera general algunas leyes relativas a las normas de tránsito, las responsabilidades ciudadanas, el ámbito familiar y el contexto escolar de los hijos. Una madre costarricense lo explicó así:

"... cuando llegué aquí [los Estados Unidos], tenía mucho miedo de salir a la calle porque yo no tengo documentos legales y como no conozco las leyes de este país, todo me daba miedo. Pensaba que si tiraba basura en la calle, me pasaba un semáforo o si no cuidaba bien a mis hijos me podían acusar de algún delito..."

En las conversaciones cotidianas que tenían con sus familiares y amistades, las participantes conocieron gradualmente las leyes con la finalidad de evitar aquellos actos que pusieran en riesgo su permanencia en los Estados Unidos. Del mismo modo, se fueron adaptando a las normas de convivencia social. Aunque la mayoría de ellas no han establecido un contacto cercano con ciudadanos norteamericanos, ha sido importante aprender a relacionarse respetuosa y cortésmente, sobre todo con las personas que trabajan en sus centros de trabajo y con el personal de la escuela donde estudian sus hijos. Una madre mexicana comentó:

"... si aprendes a convivir con las personas que viven en este país, ya sean americanos, asiáticos, árabes, latinos o quien sea, te evitas muchos problemas. Si no haces cosas que estén fuera de lugar, la gente no te va a decir que eres un desadaptado social..."

Un proceso importante de adaptación es aquel que incluye las actividades cotidianas a realizar una vez que inmigran a los Estados Unidos. Las participantes comentaron sobre las dificultades que tuvieron al comienzo de su estadía con lo referente al transporte público, para ubicar las tiendas donde comprar alimentos y demás enseres para la familia como así también el sitio donde tienen que pagar las cuentas de los servicios y las otras múltiples actividades que desempeña cualquier madre. Por otra parte, en mayor o menor medida, los inmigrantes y sus familias tienden a adaptarse a las costumbres y tradiciones propias del lugar de su nueva residen- cia. Para las madres que participaron en este estudio, no hablar con fluidez el inglés ha limitado en gran medida la adquisición y participación en las celebraciones norteamericanas. La gran mayoría de ellas comentó que continúan celebrando las tradiciones que aprendieron en sus países de origen. Particularmente, las madres mexicanas son quienes por la cercanía geográfica y la gran influencia de las costumbres mexicanas en el estado de Texas, preservan más sus celebraciones tanto religiosas como civiles.

La última categoría en este dominio está relacionada con la convivencia con los ciudadanos norteamericanos y otros grupos minoritarios. Las dificultades con el idioma inglés nuevamente son un gran obstáculo en las relaciones interpersonales con los ciudadanos norteamericanos y con la gran cantidad de otros grupos minoritarios. Debido a que sus hijos asisten a escuelas donde aproximadamente el 80\% de los estudiantes son de origen latinoamericano, las oportunidades de convivir con otros grupos étnicos es muy escasa. Solamente la madre que tiene licenciatura y quien tiene mayor fluidez en el idioma inglés nos comentó acerca de las relaciones interpersonales que ha establecido en su vecindario.

"... Ahora me llevo con una vecina que es de Siria y otras dos que son de aquí [Estados Unidos]. Mi mejor amiga es la señora de Siria porque como su hija es casi de la misma edad que mi hija nos juntamos más seguido por las tardes sobre todo a cocinar. Como su esposo trabaja todo el día, me llama mucho a que vayamos a su casa. Preparamos la comida, mientras las niñas están jugando y en la convivencia con ellas me cuenta de su país y de la familia que se quedó allá...”

\section{Expectativas}

Todas las participantes manifestaron que la principal razón para abandonar sus países de origen fue la situación económica tan precaria en la que vivían. La escasez de fuentes de trabajo o un empleo mal remunerado les permitía, cuando era posible, cubrir únicamente sus necesidades básicas. Una de sus princi- 
pales expectativas al inmigrar a los Estados Unidos era cambiar radicalmente esta situación de penuria y brindar a sus hijos condiciones de vida diferentes a las que ellos tuvieron en su infancia y adolescencia.

"... Yo sentí bien bonito la primera vez que recibí mi primer cheque. Aunque me costó mucho conseguir ese trabajo y yo no estaba acostumbrada a dejar mi casa para irme a trabajar al restaurante cuando recibí ese dinero supe que nos iba a ir muy bien. Ese dinero lo uso principalmente para mis hijos y cuando me va bien en el restaurante me queda algo para mandárselo a mis papás..."

Junto con la oportunidad de conseguir mejores condiciones laborales se presenta el sueño de un futuro más prometedor para sus hijos por medio de la educación que reciben los niños y jóvenes en los Estados Unidos. Todas las participantes se refirieron a esta situación como otra de sus principales expectativas. Por medio de otros inmigrantes que habían regresado a sus países de origen, estas madres conocieron los beneficios educativos que reciben los hijos de inmigrantes. Aun cuando sus padres llegan a los Estados Unidos en condición de indocumentados, los niños y adolescentes pueden ser inscriptos en una escuela primaria, secundaria, preparatoria e incluso en varias universidades. Asimismo, los hijos de las participantes que nacieron en los Estados Unidos podrán terminar la universidad y continuar si así lo desean con estudios de posgrado. Una madre mexicana comentó al respecto:

“... para mí, lo más importante es que mis hijas lleguen a la universidad y terminen una carrera. El día que eso suceda sabré que todos nuestros sacrificios y lo que dejamos en México valió la pena, que el esfuerzo de su papá por estar trabajando ya tiene su fruto y ellas tendrán una vida distinta a la que nos tocó vivir..."

En el momento que se realizaron las entrevistas, se estaban gestionando en el Congreso norteamericano una serie de iniciativas para una futura regularización de la situación migratoria de millones de personas que habían llegado a los Estados Unidos de manera indocumentada. Otra de las expectativas que manifestaron la mayoría de las participantes tiene que ver precisamente con esta condición. Particularmente, cuando los hijos no nacieron en los Estados Unidos y cruzaron la frontera de manera ilegal, es prioritario para sus madres arreglar el proceso migratorio de todos los miembros de la familia. El contar con un estatus legal cambia drásticamente la dinámica familiar porque libera a los padres de la preocupación de ser deportados en algún momento y les da a los hijos la seguridad de que sus papás los podrán acompañar durante su crecimiento

\section{Adversidades}

Todas las participantes coincidieron en que la principal adversidad en su proceso de aculturación ha sido el idioma. Doce participantes provienen de un medio rural donde no era común aprender un segundo idioma. Al llegar a los Estados Unidos se enfrentaron con la barrera del idioma, lo cual repercutió directamente en sus actividades parentales. Describieron dos situaciones muy particulares: por una parte, algunas madres tenían a sus hijos en una escuela donde su maestra hablaba español. Con ella se podían acercar de la misma forma que lo hacían en sus países de origen para preguntarle sobre el desempeño académico de sus hijos y diversas situaciones que se iban presentando en el ciclo escolar. Por el contrario, cuatro de las participantes comentaron que la maestra de sus hijos no hablaba español, lo cual dificultaba conocer con exactitud su rendimiento académico. Estas madres tenían muchos problemas para ayudar a sus hijos en las tareas que les dejaban y se involucraban muy poco en las actividades escolares. Una madre ecuatoriana que estaba en esta situación comentó:

“... cuando supe que la maestra de mi niña no hablaba español supe que iba a ser un año muy complicado. Aunque entiendo algo de inglés, no puedo decirle [a la maestra] lo que yo quiero, ni preguntarle mis dudas. Cuando me urge hablar con ella, le pido a una amiga que sabe bien inglés que me ayude a ir a hablar con la maestra, pero no es lo mismo porque la maestra se desespera y es muy cor- 
tante con nosotras..."

La segunda adversidad se refiere al hecho de haber dejado a sus familiares en su país de origen. Aunque ya llevaban varios años viviendo en los Estados Unidos, con mayor o menor intensidad, las madres nos hablaron de la tristeza que sienten ante la ausencia de sus familiares y amistades. Este sentimiento llega en algunos momentos a obstaculizar su labor como madres al no prestarles la suficiente atención a sus hijos o mostrarse más autoritarias con ellos. A este respecto, una madre salvadoreña comentó:

"... hay días que recuerdo que hay una fiesta en mi pueblo o es el cumpleaños de alguien de mi familia y me siento muy triste. Esos días me enojo de todo y si mis hijos me piden algo los ignoro o no les doy las cosas. Eso me hace sentir todavía más triste porque sé que no tengo que regañarlos nada más porque me siento triste..."

Las participantes describieron también las dificultades que tienen que enfrentar debido a su estatus migratorio. Con excepción de una madre, todas las participantes entraron ilegalmente a los Estados Unidos. Esta situación las coloca en una condición muy vulnerable y crea una preocupación constante con respecto a su futuro y sobre todo el de su familia. Aunque su labor parental no se ve afectada directamente por la falta de documentos migratorios, la posibilidad de continuar con sus estudios y el acceso a conseguir un mejor puesto de trabajo es sumamente complicado. Una madre mexicana se refirió a ello de la siguiente manera:

"... Para mí es muy difícil conseguir un mejor trabajo porque en todos lados te piden papeles, si no tienes permiso para trabajar te quieren pagar lo mínimo y tienes que trabajar haciéndola de todo. Esos trabajos son muy pesados y el horario es bien difícil..."

Finalmente, dos de las participantes comentaron ampliamente lo difícil que fue para ellas dejar a alguno de sus hijos en su país de origen. En ambos casos, la razón fue la falta de recursos económicos para realizar el viaje a los Estados Unidos. Para una de ellas en lo particular, los años que lleva separada de él han sido una pesadilla. Al principio parecía que el tiempo de espera no sería mucho; sin embargo, la falta de dinero y sobre todo lo peligroso que es atravesar México para después cruzar la frontera determinaron como imposible que sus hijos se reúnan con ellas. Esta situación provoca tristeza, frustración, y genera al mismo tiempo otras ideas que determinan negativamente su percepción de lo que significa ser una "buena" madre.

\section{Beneficios}

A pesar de los muchos obstáculos a los que se tienen que enfrentar, todas las participantes estuvieron de acuerdo al señalar contundentemente que valió la pena haber inmigrado a los Estados Unidos. El punto de mayor coincidencia está relacionado con la educación de los hijos. Como se mencionó anteriormente, solamente tres participantes tienen estudios de nivel medio superior. En sus países de origen, la mayoría de estas inmigrantes no tuvieron acceso a un buen nivel educativo que les permitiera contar con un bagaje de conocimientos. Al encontrarse en los Estados Unidos han visto la calidad educativa que están recibiendo sus hijos y la pueden comparar con todas las deficiencias que ellas padecieron. Aunque son conscientes que muy probablemente no podrán continuar con sus estudios, el saber que sus hijos tendrán un porvenir más esperanzador es la mejor prueba de que valió la pena dejar sus países de origen.

“... la educación que están recibiendo mis hijos en este país es un sueño para mí. Yo nunca imaginé que un día mis hijos podrían llegar a la universidad. En el rancho en el que viví apenas había una primaria y ahora veo la escuela de mis hijos y me siento tan contenta porque ellos pueden estudiar y llegar muy alto en sus estudios..."

Un segundo beneficio que ha tenido un impacto directo en su desempeño como madres es la información que han recibido por medio de cursos, talleres y pláticas impartidos por profesionales de distintas disciplinas. En las escuelas donde estudian sus hijos, se ofrecen regularmente este tipo de actividades a través de las cuales las madres conocen me- 
jores estrategias parentales y estilos de crianza más adecuados. Debido a que la mayoría de las madres latinoamericanas no domina el idioma inglés, la gran ventaja de estos cursos es que son impartidos en español y sin ningún costo. Además de recibir información profesional, en estos cursos se comentan aspectos legales los cuales las madres tienen que saber para evitar incurrir en una práctica que pueda tener como resultado la pérdida de la custodia de sus mismos hijos. Una madre mexicana lo ilustró de la siguiente manera:

“... como uno viene del rancho está acostumbrada a solo regañar a los hijos y si no se portan bien pues unas nalgadas para que entiendan. Pero aquí [en los Estados Unidos] hay que tener mucho cuidado porque con tantito te quieren quitar a los hijos. Eso lo aprendí en las clases que dan a los papás. Vienen profesoras a hablarnos de cómo hacerle para no regañar tanto a los hijos y cómo ayudarles a hacer la tarea y lo que nos piden las maestras..."

Para este grupo de inmigrantes, quienes en su mayoría tenían en sus países de origen un nivel socioeconómico muy bajo, las oportunidades de empleo que han encontrado en los Estados Unidos han cambiado radicalmente su estilo de vida. El tener acceso a un empleo bien remunerado ha tenido un impacto positivo en la familia al proporcionar a sus hijos y a ellos mismos una mejor alimentación, acceso a los servicios de salud, una educación de buen nivel para sus hijos y la posibilidad de momentos de esparcimiento en común. En aquellas familias en las cuales la madre también trabaja, los ingresos les permiten a su vez contar con una casa más amplia y tener un medio de transporte propio.

Otros dos beneficios muy importantes tienen que ver con la posibilidad de volver a estar juntos como familia y la mejor atención a los hijos. Nueve de las participantes no emigraron al mismo tiempo con su esposo. En la mayoría de los casos, ellos ya habían emigrado y llevaban algunos meses o incluso años viviendo solos en los Estados Unidos. Una vez que emigraron y se reunieron con su esposo pudieron disfrutar de su compañía y comenzar una nueva etapa familiar. Esta reunificación junto con la información que reciben en los Estados Unidos, las pláticas informales con otras latinoamericanas, la influencia de los otros grupos étnicos y su propio proceso de aculturación les permite tener mejores recursos y estrategias para brindar una atención más apropiada a sus hijos. Esta atención no solamente se traduce en proveerles objetos materiales sino y sobre todo la compañía, comunicación y expresiones de afecto tan intensas como las que saben brindar las madres latinoamericanas. Una de las participantes salvadoreñas lo relató de la siguiente manera:

"... Yo todos los días les digo a mis hijos que los amo, a cada ratito trato de decírselos, los abrazo, los apapacho. Cuando llegan de la escuela lo primero que hago es preguntarles cómo les fue, qué hicieron. Por las tardes cuando tenemos tiempo nos ponemos a ver la tele o salimos al parque. Así es como yo les demuestro mi atención a mis hijos, diciéndoles lo mucho que los quiero..."

Un beneficio más que describieron las participantes fue la condición de bilingües que tienen sus hijos. Al estar estudiando en escuelas donde se enseña solamente en inglés o en aquellas que tienen programas bilingües, los niños comienzan a aprender el idioma de manera natural. Al terminar la primaria la inmensa mayoría de los niños ya puede hablar de manera fluida en inglés y el español lo continúan practicando en sus hogares. Finalmente, una situación muy particular fue descrita por algunas participantes como un beneficio. Los hijos que nacieron en los Estados Unidos adquieren la doble nacionalidad. De esta manera, cuando llegan a la adolescencia pueden viajar a los países de origen de sus padres a visitar a sus abuelos, tíos, primos y demás familiares. Las madres que se encuentran en esta condición comentaron que a través de ese contacto, ellas se sienten más cerca de sus familias.

\section{Recursos}

El principal recurso que expresaron las madres fue la ayuda que reciben del gobierno 
norteamericano, tanto del federal como del estatal. Por ejemplo, las madres que se embarazaron en los Estados Unidos recibieron múltiples beneficios durante esta etapa y después de que nacieron sus hijos. En el estado de Texas, las mujeres embarazadas y de escasos recursos tienen la oportunidad de recibir ayuda médica gratuita durante todo el embarazo y al momento del nacimiento. Al nacer sus hijos, reciben también de forma gratuita atención pediátrica y hay un seguimiento médico para las madres con la finalidad de descartar alguna complicación durante su recuperación. Esta situación la describió una madre ecuatoriana:

“... Desde que nació mi hija nunca he batallado con el seguro médico. Gracias al seguro recibió sus primeras vacunas, la llevábamos con el doctor y las estampillas para comprar alimentos eran de mucha ayuda cuando comenzó a comer... También la llevamos al dentista y yo iba a las pláticas de la lactancia para saber cómo darle de comer y ahí [en el centro médico] me apoyaban mucho..."

Esta ayuda gubernamental volvió a ser de suma importancia cuando los hijos entraron a la escuela. Además de recibir una educación gratuita, las familias de bajos recursos reciben distintas ayudas durante el ciclo escolar para recibir gratuitamente el desayuno escolar, apoyo económico para la compra de algunos artículos escolares y el transporte. Una madre peruana habló de la importancia de este apoyo de la siguiente manera:

"... Cuando mis hijos entraron en la primaria fue de mucha ayuda que ellos no tuvieron que pagar nunca el desayuno que les daban en la escuela. Como era muy bueno el desayuno, yo ya no tenía que hacer ningún gasto para mandarles algo durante la maก̃ana..."

Junto con la ayuda gubernamental, las participantes enfatizaron que el conocimiento que reciben proveniente de distintos medios también es un recurso muy importante para la crianza de sus hijos. Este conocimiento se traduce principalmente en estrategias más eficaces de involucramiento emocional. Si bien todas las participantes describieron amplia- mente la cercanía y el amor que sienten para sus hijos, por medio del conocimiento formal e informal al que tienen acceso, ellas han sabido potencializar el desarrollo emocional de sus hijos. Por consiguiente, este recurso impacta directamente en su desempeño como madres.

La última categoría incluida en este dominio se refiere a las redes de apoyo social que han ido conformando los propios latinoamericanos. Unas de las más importantes son las organizaciones civiles latinoamericanas. Estas se diversifican en distintas áreas incluyendo las culturales, comerciales, deportivas, jurídicas, médicas y de esparcimiento en general. A ellas, los inmigrantes latinoamericanos acuden para recibir múltiples servicios como lo son las asesorías, tratamientos e incluso capacitación para convertirse ellos mismos en promotores de estas organizaciones. Igualmente, las iglesias en sus diferentes denominaciones son un lugar de encuentro para los latinoamericanos tanto los inmigrantes como aquellos que llevan muchos años viviendo en los Estados Unidos. Además de encontrar un lugar seguro para convivir, las iglesias ofrecen diversos servicios con la finalidad de brindar información a la comunidad latinoamericana y de esta manera hacer más fácil su adaptación.

\section{Discusión}

El proceso de aculturación está lleno de desafíos y al mismo tiempo de múltiples beneficios para los inmigrantes latinoamericanos y primordialmente para sus hijos. El estudio realizado permitió conocer de manera puntual el impacto que tiene la aculturación en el área del funcionamiento parental. El primer factor que se consideró fue el proceso de adaptación el cual conlleva para un gran número de padres latinoamericanos un sinfín de obstáculos y retos. Diversos estudios han demostrado que el involucramiento y el desempeño parental de los inmigrantes latinoamericanos pueden cambiar con el tiempo dependiendo de las actitudes, creencias y comportamientos que van adoptando (Borns- 
tein \& Cote, 2004; Dumka, Roosa \& Jackson, 1997). Una vez que las inmigrantes fueron conociendo paulatinamente las leyes y normas de convivencia social imperantes en la cultura norteamericana, ellas hicieron importantes cambios en su comportamiento con la finalidad de no violar alguna ley y tener al mismo tiempo una adaptación socialmente aceptable. Especialmente en lo relativo a las leyes que regulan el trato que se les debe dar a los niños en los Estados Unidos, las participantes nos comentaron que fueron de gran ayuda las pláticas que recibieron en las escuelas así como las charlas informales con sus amigas.

Por otra parte, el modelo dimensional describe los distintos niveles de aculturación por los que pasan los inmigrantes. En este proceso, las estrategias de aculturación surgen como actitudes psicológicas que incluyen diversos elementos entre los que destacan los valores, las creencias, el uso del idioma, las percepciones y las conductas de los inmigrantes. Estas estrategias son consideradas como recursos internos que posee cada inmigrante con la finalidad de emplearlas en las distintas etapas de su proceso de adaptación. Por medio de estas estrategias, los inmigrantes enfrentan los retos que trae consigo su estadía en un nuevo país así como los cambios que van llevando a cabo a nivel individual y familiar.

En lo que respecta a las tradiciones y costumbres familiares, todas las participantes mostraron un apego a las creencias y valores que aprendieron en sus países de origen. Con excepción de la conmemoración del 4 de julio (día de la independencia), las participantes no mencionaron otra festividad que hayan adquirido y celebren en familia. Según Berry (1980), la estrategia de aculturación llamada separación ocurre cuando los migrantes mantienen su cultura de origen y al mismo tiempo evitan interactuar con otros grupos étnicos, principalmente con las personas nativas del país en el cual ahora están residiendo. Una posible explicación de esta falta de contacto con otras madres nativas o pertenecientes a otros grupos étnicos es la falta de fluidez en el idioma inglés. Como consecuencia de ello, las participantes de este estudio preferían convivir con otras madres latinoamericanas y realizar sus actividades cotidianas en lugares donde la comunidad latinoamericana suele reunirse.

Debido a la falta de preparación académica, recursos económicos y su estatus migratorio, los inmigrantes latinoamericanos se enfrentan a un sinfín de adversidades especialmente durante los primeros meses. Diversos estudios han documentado como la discriminación, la barrera del idioma, el miedo a la deportación, la explotación laboral, conflictos intergeneracionales, la deserción escolar, la promulgación de leyes antiinmigrantes, bajos salarios y la falta de fluidez en el inglés son importantes factores de riesgo para los inmigrantes latinoamericanos (e.g., Araujo \& Borrells, 2006; Caplan, 2007; Cavazos-Rehg, Zayas \& Spitznagel, 2007; García \& Lindgren, 2009; Parra-Cardona, Bulock, Imig, Villaruel \& Gold, 2006). Como lo comentaron las participantes, el haber dejado a sus familias provocó una constante nostalgia y tristeza. Además de enfrentar esta condición emocional, otra gran adversidad que las acompaña durante muchos años es la falta de fluidez en el idioma inglés. Las repercusiones negativas que tiene en su desempeño como madres se hacen más evidentes cuando sus hijos asisten a escuelas que no son bilingües. En estas instituciones, la mayoría de las maestras no saben español y toda la información que les proporcionan a las madres se encuentra en inglés.

A pesar de todas estas adversidades, su nueva vida en los Estados Unidos les ha dado múltiples beneficios. Uno de los hallazgos más importantes es el consenso hecho por todas las participantes al expresar que valió la pena emigrar. En lo que respecta a los hijos, los principales beneficios están relacionados con el nivel educativo que pueden alcanzar, el llegar a ser bilingües y el apoyo gubernamental que reciben. Particularmente, los hijos que nacieron en los Estados Unidos tienen todos los derechos de un ciudadano norteamericano y a diferencia de los niños inmigrantes, ellos cuentan con la oportunidad de visitar a las familias de sus padres que se encuentran 
viviendo en sus países de origen. Otros de los beneficios que ha traído consigo el proceso de aculturación es la instrucción ya sea a través de las pláticas o talleres que reciben en las escuelas de sus hijo o por medio de charlas informales con otras madres latinoamericanas con respecto a la mejor atención que les pueden brindar a sus hijos. Esta información la han empleado con el objetivo de lograr un mayor involucramiento con sus hijos así como potencializar sus capacidades como madres. El convivir primordialmente con otras madres latinoamericanas les ha permitido no solamente mantener sus raíces y sentirse más acogidas, sino sobre todo recibir el apoyo necesario para familiarizarse con las normas y tradiciones propias de los Estados Unidos. En otro estudio con población latinoamericana, los resultados mostraron que las madres menos apegadas a la cultura norteamericana y quienes acababan de inmigrar se involucraban más en las actividades académicas de sus hijos y estaban más pendientes de su comportamiento en comparación con las madres latinoamericanas con niveles más altos de aculturación (Dinh, Roosa, Tein \& Lopez, 2002).

Por otra parte, a diferencia de las condiciones tan precarias en las que vivían en sus países de origen, su situación económica ha cambiado radicalmente. Después de algunos meses de trabajo, tanto ellas como sus esposos han conseguido una mejor retribución económica la cual les ha permito gozar de más recursos económicos, los cuales emplean no solamente para el esparcimiento sino también para el bienestar integral de los miembros de la familia. En un estudio que incluyó la participación de padres viviendo en vecindarios localizados en zonas de bajos recursos económicos, los resultados mostraron que estos padres tienen percepciones más pobres de su nivel de eficacia así como menos involucramiento parental y capacidad para monitorear el comportamiento de sus hijos (Shumow \& Lomax, 2002). El aumento de sus recursos económicos ha cambiado su percepción parental al descubrir que de la misma manera que pueden desempeñarse adecuadamente en su lugar de trabajo, lo pueden hacer en la crianza de sus hijos.

Por distintas circunstancias, algunas de las participantes en este estudio no emigraron al mismo tiempo que sus esposos. Después de algunos meses e incluso años, todas pudieron reunirse nuevamente con ellos. Este ha sido un beneficio determinante para el bienestar de la familia y su mejor desempeño como madres. El apoyo de sus parejas ha sido muy importante para que ellas puedan adaptarse de la mejor manera al estilo de vida norteamericano. Gracias a esta ayuda, han dejado a un lado las principales preocupaciones inherentes a cualquier inmigrante para concentrarse de mejor manera en la crianza de los hijos. Estos hallazgos son consistentes con otras investigaciones que han enfatizado el impacto del proceso de aculturación en el desarrollo social de los hijos y la importancia de la convivencia familiar (Crouter, Davis, Updegraff, Delgado \& Fortner, 2006).

Antes de tomar la decisión de emigrar a los Estados Unidos, las participantes tenían como principales expectativas que sus hijos tuvieran la educación que ellas no recibieron así como conseguir un trabajo bien remunerado que trajera por ende un mejor nivel socioeconómico. En distinto grado, todas las participantes han conseguido satisfacer estas dos expectativas. Por el contrario, una de sus expectativas más importantes al momento de la entrevista era la posibilidad de arreglar su situación migratoria. Como se mencionó anteriormente, la falta de documentos para permanecer legalmente en el país trae como consecuencia muchas restricciones y al mismo tiempo un miedo constante a ser deportadas. Esta fue la razón por la cual comentaron que el encontrar una solución a su situación migratoria se ha convertido en su principal expectativa.

Como se ha visto en los estudios que abordan el fenómeno de la migración, los recursos que un inmigrante puede conseguir son determinantes para su proceso de aculturación. De manera particular, las madres de hijos nacidos en los Estados Unidos gozan de 
múltiples beneficios porque el gobierno les brinda distintos tipos de ayuda en lo que se refiere a salud, educación, alimentación y protección de los niños. Además de gozar del apoyo gubernamental, diversas organizaciones civiles y religiosas han conformado una red de ayuda a los inmigrantes latinoamericanos. En las ciudades con gran afluencia de latinoamericanos, los inmigrantes tienen la oportunidad de recibir apoyo material que se traduce en enseres para el hogar, alimentos, ropa, útiles escolares así como algunos aparatos electrónicos de segunda mano. Otro tipo de ayuda que es muy importante para el bienestar de la familia tiene que ver con los servicios médicos, las asesorías de tipo legal, la consejería psicológica, la guía espiritual y las actividades extraescolares de sus hijos. Tanto el apoyo institucional como aquel que proviene de familiares y vecinos es un gran aliciente cuando vienen crisis económicas, enfermedades o cambios intempestivos en la dinámica familiar. De acuerdo con Gonzales, Knight, Morgan-Lopez, Saenz \& Sirolli (2001), los inmigrantes latinoamericanos con menores niveles de aculturación tienen regularmente poca instrucción académica lo cual los lleva a emplearse en trabajos poco remunerados; sin embargo, a pesar de estas carencias, estos inmigrantes tienden a ser sumamente solidarios con otros latinoamericanos ofreciendo todo tipo de apoyo emocional y económico.

Con la finalidad de sintetizar la información proporcionada por las participantes con respecto a la percepción que tienen de sus capacidades y recursos como madres, es importante enfatizar el amor, comprensión, interés, compromiso, solicitud, entrega, atención, esmero y cariño con el que educan a sus hijos. El vínculo que mantienen con ellos a lo largo de su vida está caracterizado por un profundo afecto. A su vez, los innumerables sacrificios y su constante esfuerzo por buscar el bienestar y la felicidad de sus hijos es uno de los rasgos más importante que distingue a las madres latinoamericanas viviendo en los Estados Unidos. Además de sus propias capacidades, los recursos que han encontrado en este país han sido determi- nando para desempeñar su labor maternal. Las participantes destacaron la ayuda que reciben del gobierno norteamericano, el conocimiento que han adquirido a través de distintos cursos y talleres, la estabilidad económica que han encontrado como resultado de un trabajo bien remunerado y las redes de apoyo que han establecido con otras familias migrantes.

Una vez hecho el análisis de los datos cualitativos se encontraron varias limitaciones en la investigación realizada. La primera de ellas tiene que ver con la diversidad del país de origen de las participantes. La muestra solamente incluyó a madres provenientes de cinco países latinoamericanos. Como se ha mencionado en varios meta-análisis, es prioritario identificar las diferencias que tienen entre sí los diversos grupos latinoamericanos de acuerdo a su país de origen. Esto haría posible conocer con exactitud las características particulares de cada subgrupo y por ende, brindarles mejores servicios. Otra limitación estuvo relacionada con las características de la muestra la cual fue recolectada en una ciudad y en un estado norteamericano con una numerosa población latinoamericana. Estudios posteriores deberían examinar el impacto que el proceso de aculturación tiene para los inmigrantes viviendo en zonas donde los latinoamericanos constituyen una minoría. Sería de gran interés comparar los hallazgos en investigaciones tan opuestas con la finalidad de contrastar las desventajas y beneficios que se encuentran en ambos contextos. Por otra parte, todas las participantes tenían un estatus de indocumentadas el cual afectaba directamente su involucramiento y desempeño como madres. Los estudios que se lleven a cabo en el futuro deberían incluir algunas madres que hayan inmigrado legalmente o quienes hayan conseguido regularizar su estatus migratorio. La información que se recolecte de estas madres daría una perspectiva complementaria con respecto al involucramiento y funcionamiento parental que se puede alcanzar al verse libres del constante miedo a la deportación. Finalmente, sería muy enriquecedor incluir la medición cuantitativa del nivel de aculturación de cada par- 
ticipante. Es un elemento reiterativo en los estudios sobre migración y en las teorías que explican el proceso de la aculturación la importancia de identificar en qué punto del continuum se encuentra cada inmigrante. Los hallazgos empíricos reportan importantes diferencias entre los inmigrantes que han abandonado radicalmente los lazos con sus raíces étnicas en comparación con aquellos que aun viviendo en los Estados Unidos siguen con sus actividades cotidianas, sus relaciones interpersonales y su forma de pensar como si estuvieran todavía viviendo en su país de origen.

\section{Cuadro 1}

Análisis cualitativo de la experiencia de ser madre e inmigrante

\begin{tabular}{ll}
\hline \multicolumn{1}{c}{ Dominio/Categoría } & Frecuencia \\
\hline Adaptación & \\
Conocimiento de las leyes norteamericanas & Típica \\
Normas de convivencia social & General \\
Actividades cotidianas & General \\
Costumbres y tradiciones & Divergente \\
Relaciones interpersonales & Típica \\
Expectativas & \\
Mejores condiciones laborales & General \\
Educación de calidad para sus hijos & General \\
Regularización de su situación migratoria & General \\
Adversidades & \\
Idioma inglés & General \\
Separación de la familia extensa & Típica \\
Estatus migratorio & General \\
Separación de un hijo & Divergente \\
Beneficios & \\
Educación de los hijos & General \\
Información profesional & Típica \\
Empleo remunerado & Típica \\
Reunificación familiar & Típica \\
Mejor atención a los hijos & General \\
Fluidez de los hijos en ambos idiomas & General \\
Hijos nacidos en los Estados Unidos & Divergente \\
Ayuda del gobierno norteamericano & \\
Conocimiento profesional & General \\
Redes de apoyo social para latinoamericanos & Divergente \\
\hline & Típica \\
\hline
\end{tabular}




\section{Referencias bibliográficas}

Araujo, B. \& Borrells, L. (2006). Understanding the link between discrimination, mental health outcomes and life chances among Latinos. Hispanic Journal of Behavioral Sciences, 28, 245-266.

Ardelt, M. \& Eccles, J. (2001). Effects of mother's parental efficacy beliefs and promotive parenting strategies on inner-city youth. Journal of Family Issues, 22(8), 944-972.

Bandura, A. (1986). Social foundations of thought and action: A social cognitive theory. Englewood Cliffs, NJ: Prentice-Hall.

Bandura, A. (1977). Self-efficacy: Toward a unifying theory of behavioral change. Psychological Review, 84(2), 191-215.

Bandura, A. (2012). On the functional properties of perceived self-efficacy revisited. Journal of Management, 38(1), 9-44.

Bacallao, M.L. \& Smokowski, P.R. (2007). The costs of getting ahead: Mexican family system changes after immigration. Family Relations, 56, 52-66.

Berry, J.W. (1980). Acculturation as varieties of adaptation. En A.M. Padilla (Ed.), Acculturation: Theory, models and some new findings (pp. 9-25). Boulder, CO: Westview.

Berry, J.W. (1997). Immigration, acculturation, and adaptation. Applied Psychology, 46, 5-34.

Berry, J.W. (2001). A psychology of immigration. Journal of Social Issues, 57(3), 615-631.

Bornstein, M.H. \& Cote, L.R. (2004). Mothers' parenting cognitions in cultures of origin, acculturating cultures, and cultures of destination. Child Development, 75(1), 221-235.

Bourhis, R. \& Monteuil, A. (2001). Majority acculturation orientations toward "valued" and "devalued" immigrants. Journal of Cross-cultural Psychology, 32(6), 698-719.

Brown, C., Langille, D., Tanner, J. \& Asbridge, M. (2014). Health-compromising behaviors among a multi-ethnic sample of Canadian high school students: Risk-enhancing effects of discrimination and acculturation. Journal of Ethnicity in Substance Abuse, 13(2), 158178.

Caplan, S. (2007). Latinos, acculturation, and acculturative stress: A dimensional concept analysis. Policy, Politics, and Nursing Practice, 8, 93-106.

Cavazos-Rehg, P.A., Zayas, L.H. \& Spitznagel, E.L. (2007). Legal status, emotional well-being and subjective health status of Latino immigrants. Journal of the National Medical Association, 99, 1126-1131.

Crouter, A.C., Davis, K.D., Updegraff, K., Delgado, M. \& Fortner, M. (2006). Mexican American fathers' occupational conditions: Links to family members' psychological adjustment. Journal of Marriage and Family, 68, 843-858.

Dinh, K.T., Roosa, M.W., Tein, J.Y. \& Lopez, V. A. (2002). The relationship between acculturation and problem behavior proneness in a Hispanic youth sample: A longitudinal mediation model. Journal of Abnormal Child Psychology, 30(3), 295-309.

Dumka, L.E., Roosa, M.W. \& Jackson, K.M. (1997). Risk, conflict, mothers' parenting, and children's adjustment in low-income, Mexican immigrant, and Mexican American families. Journal of Marriage and the Family, 59, 309323.

Finley, G.E. \& Schwartz, S.J. (2004). The father involvement and nurturant fathering scales: Retrospective measures for adolescent and adult children. Educational and Psychological Measurement, 64, 143-164

Fontes, L.A. (2002). Child discipline and physical abuse in immigrant Latino families: Reducing violence and misunderstandings. Journal of Counseling and Development, 80, 31-40.

García, C. \& Lindgren, S. (2009). Life grows between the rocks: Latino adolescent's and parents' perspective on mental health stressors. Research in Nursing Health, 32(2), 148-162. 
Gonzales, N.A., Knight, G.P., Morgan-Lopez, A., Saenz, D.S. \& Sirolli, A. (2001). Acculturation, enculturation and the mental health of Latino youths: An integration and critique of the literature. En J.M. Contreras, K.A. Kerns \& A.M. Neal-Barnett (Eds.), Latino children and families in the United States (pp. 45-74). Westport, CT: Greenwood.

González-Rábago, Y. (2014). Los procesos de integración de personas inmigrantes: Límites y nuevas aportaciones para un estudio más integral. Athenea Digital, 14(1), 195-220.

Gutmann, M. (2003). Changing men and masculinities in Latin America. Durham, NC: Duke University Press.

Hill, C.E., Thompson, B.J. \& Williams, E.N. (1997). A guide to conducting consensual qualitative research. The Counseling Psychologist, 25, 517-572.

Jones, T.L. \& Prinz, R.J. (2005). Potential roles of parental self-efficacy in parent and child adjustment: A review. Clinical Psychology Review, 25, 341-363.

LaFramboise, T.D., Coleman, H.L.K. \& Gerton, J. (1993). Psychological impact of biculturalism: Evidence and theory. Psychological Bulletin, 114, 395-412.

Lawton, K.E. \& Gerdes, A.C. (2014). Acculturation and Latino adolescent mental health: Integration of individual, environmental, and family influences. Clinical Child and Family Psychology Review, 17(4), 385-398.

Lee, J., Yu, H. \& Choi, S. (2012). The influences of parental acceptance and parental control on school adjustment and academic achievement for South Korean children: The mediation role of self-regulation. Asia Pacific Education Review, 13(2), 227-237.
Marsiglia, F.F., Nagoshi, J.L., Parsai, M. \& Castro, F.G. (2014). The effects of parental acculturation and parenting practices on the substance use of Mexican-heritage adolescents from Southwestern Mexican neighborhoods. Journal of Ethnicity in Substance Abuse, 13(3), 288311.

Parra-Cardona, J.R., Bulock, L.A., Imig, D.R., Villaruel, F.A. \& Gold, S.J. (2006). Trabajando duro todos los días: Learning from the experiences of Mexican-origin inmigrant families. Family Relations, 55, 361-375.

Pew Research Center (2008). U.S. Population Projections: 2005-2050. Washington, DC: Pew Reseach Center.

Redfield, R., Linton, R. \& Herskovits, M.J. (1936). Memorandum for the study of acculturation. American Anthropologist, 38, 149-152.

Schleider, J.L., Vélez, C.E., Krause, E.D. \& Gillham, J. (2014). Perceived psychological control and anxiety in early adolescents: The mediating role of attributional style. Cognitive Therapy and Research, 38(1), 71-81.

Shumow, L. \& Lomax, R. (2002). Parental efficacy: Predictor of parenting behavior and adolescent outcomes. Parenting: Science and Practice, 2, 127-150.

Tran, D.T., Jorm, L., Johnson, M., Bambrick, H. \& Lujic, S. (2015). Effects of acculturation on lifestyle and health status among older Vietnamborn Australians. Asia Pacific Journal of Public Health, 27(2), 2259-2274.

Unitesd States Census Bureau. (2015). Statistical Abstract of the United States, 2015. Washington, D.C: U.S. Dept. Commerce.

Umaña-Taylor, A.J., Bhanot, R. \& Shin, N. (2006). Ethnic identity formation during adolescence: The critical role of families. Journal of Family Issues, 27, 390-414. 
